\title{
Multidimensional assessment of interoceptive awareness (MAIA 2): psychometric properties of the Turkish version
}

\author{
๑Saliha Özpınar', @Emre Dünder ${ }^{2}$, ๑Yaşar Demir ${ }^{3}, \oplus$ Melih Akyol $^{4}$ \\ ${ }^{1}$ Alanya Alaaddin Keykubat University, School of Medicine, Department of Public Health, Antalya, Turkey \\ ${ }^{2}$ Ondokuz Mayıs University, Faculty of Science and Literature, Department of Statistics, Samsun, Turkey \\ ${ }^{3}$ Alanya Alaaddin Keykubat University, Institute of Health Sciences, Antalya, Turkey \\ ${ }^{4}$ Cumhuriyet University, School of Medicine, Department of Dermatology, Sivas, Turkey
}

Cite this article as: Özpınar S, Dünder E, Demir Y, Akyol M. Multidimensional assessment of interoceptive awareness (MAIA 2): psychometric properties of the Turkish version. J Health Sci Med 2021; 4(2): 132-136.

\begin{abstract}
Aim: Interoception, defined as the perception of internal bodily changes. The aim of this study is to evaluate the Turkish validity and reliability study of the Multidimensional Assessment of Interoceptive Awareness-2 (MAIA 2) scale developed by William Mehling et al. (2018).

Material and Method: The research is methodological. Research data was collected between April 2020 and May 2020 ( $\mathrm{n}=400)$. Forward and backward translation were used to translate the MAIA 2 into Turkish. We conducted exploratory factor analysis (EFA) and confirmatory factor analysis (CFA) to analyze the psychometrics of the MAIA 2 in a 70:30 split sample. Statistical analysis were done with R Project.

Results: The Kaiser-Meyer-Olkin value $(\mathrm{KMO}=0.910)$ was acceptable, and there exists a significant correlation structure with Bartlett's test of sphericity $(\chi 2=5134.120, p<0.001)$. We observed six factors with Horn's Parallel analysis. The Cronbach Alphas of these six factors were acceptable $(\alpha>0.60)$. Standardized loadings were positive and $>0.40$ with significant results $(\mathrm{p}<0.05)$ $(\mathrm{r}=0.71, \mathrm{p}<0.05)$. The RMSEA is nearly zero and SRMR is extremely low.

Conclusions: As a result, the Turkish version of the Multidimensional Assessment of Interoceptive Awareness Scale (MAIA 2) developed by Mehling et al. and originally in English is valid and reliable according to our results.

Keywords: Body awareness, body-mind, interoceptive awareness, validity, reliability
\end{abstract}

\section{INTRODUCTION}

While interoception is a term that has gained and still is gaining popularity in the academic literature since the start of the millennium, consensus on its meaning is as yet not fully established. What is generally agreed upon by most current scholars is that interoception is the perception of the state of the body $(1,2)$.

Interoception is a sense that provides information about the internal condition of our body-how our body is feeling on the inside. Interoception allows us to experience many body sensations such as a growling stomach, dry mouth, tense muscles or racing heart.

Although several self-report measures to assess interoceptive and/or body awareness are available, they assess only very limited aspects (e.g., negative aspects) of the concept, which may not capture the complex nature of interoceptive awareness (3). The Multidimensional
Assessment of Interoceptive Awareness (MAIA) questionnaire is designed to assess interoceptive awareness (4). The MAIA is a questionnaire able to distinguish between different interoceptive attentional styles that can be adaptive (referred to as a receptive, mindful awareness attitude according to Kabat-Zinn (5) or maladaptive (e.g., anxiety-driven) in processing interoceptive sensations to regulate emotions and behavior. Additionally, the MAIA has been used to assess changes in interoceptive awareness in interventional studies (3-6).

This article presents the adaptation into the Turkish language of the multidimensional assessment of interoceptive awareness-2 (MAIA 2) self report instrument developed by Mehling et al. (7) (2018), and the evaluation of its psychometric properties in the Turkish population. 


\section{MATERIAL AND METHOD}

All procedures were performed adhered to the ethical rules and the Helsinki Declaration of Principles. Ethical committee permission for the study was granted by Alanya Alaaddin Keykubat University Clinical Researches Ethics Committee (decision no: 20-3; date: 18.06.2020). In addition, legal permission was obtained from the hospitals to conduct the research. Informed consent was obtained from the individuals who participated in the study.

\section{Study Design, Setting and Sample}

Research data was collected between April 2020 and May 2020. The study is of a methodological type. The sample of this study includes 403 healthcare workers. For methodological research, the sample size is recommended to be at least $5-10$ times more than the number of the items of the scale $(8,9)$. The healthcare workers including physicians, dentist, nurses, midwife, health officer were evaluated as eligible to participate in the study.

All the participants were notified of the study's purpose and methods, and assured that their privacy would be protected. Once they agreed to participate by completing a written consent form, they were administered the questionnaires. Subsequently, on receiving the completed questionnaires, the research assistant checked them for missing values.

\section{Instrument}

The MAIA 2 is a self-administered instrument developed by Mehling et al. (7) (2018) to measure eight dimensions of interoceptive body awareness. It has a total of 37 items tested on a Likert scale, with six levels of ordinal response coded from 0 (never) to 5 (always). The number of items and reliability established by Cronbach's alpha $(\alpha)$, vary among the subscales: noticing ( 4 items, $\alpha=0.64$ ), Not-Distracting (6 items, $\alpha=0.74$ ), Not-Worrying (5 items, $\alpha=0.67$ ), Attention Regulation (7 items, $\alpha=0.83$ ), Emotional Awareness (5 items, $\alpha=0.79$ ), Self-Regulation ( 4 items, $\alpha=0.79$ ), Body Listening ( 3 items, $\alpha=0.80$ ) and Trusting ( 3 items, $\alpha=0.83$ ) (7).

\section{Data Analysis}

We implemented exploratory factor analysis (EFA) and confirmatory factor analysis (CFA) to analyze the scale adoption process of the MAIA 2 scale items. We split the data into two parts (70:30 ratio) for exploring and validating the dimensions of the scale. In the first phase, we carried out EFA and reliability analysis based on the sub-dimensions. Then, we validated the factors using CFA. All the statistical analysis findings were obtained with R Project (10) using two R packages; psych (11) and lavaan (12).

\section{MAIA 2 Translation into Turkish}

We adapted the MAIA 2 into Turkish using a systematic translation with the aid of a focus group. Three native Turkish speakers, fluent in English and familiar with the concepts of interoception and mindfulness, independently translated the 37 items of the English MAIA 2 version into Turkish. The three Turkish versions were discussed by these experts to ensure the conceptual equivalence of the items. The consensus version was then cognitively tested in a 1-hour focus group of ten health care worker, led by two moderators who documented suggestions and comments and audio-recorded the meeting. Using the input from these focus-group participants and moderators, several items were revised for a consensual version of the questionnaire (MAIA 2 Turkish).

\section{RESULTS}

\section{Sample Characteristics}

Regarding the age distribution of research group $34.8 \pm 8.4$ (20-62), $54.7 \%$ was female and $45.3 \%$ was male. The HCWs including physicians (29.6\%), dentist (3.9\%), nurses $(29.1 \%)$, midwife $(6.9 \%)$, health officer $(30.5 \%)$ were evaluated as eligible to participate in the study.

\section{Statistical Analysis}

We used principal axis method for the extraction process with varimax rotation approach in EFA. Since the scale items were ordinal, we considered Polychoric correlation matrix during EFA (25).

The Kaiser-Meyer-Olkin value $(\mathrm{KMO}=0.910)$ was acceptable, and there existed a significant correlation structure with Bartlett's test of sphericity $\chi 2=5134.120$, $\mathrm{p}<0.001$. We observed six factors with Horn's Parallel analysis.

Table 1 shows the rotated component matrix and communality values results for the MAIA 2 scale. Because of the low loadings in rotated component matrix, totally five items were removed. The communality values were relatively high and the remained factor loadings were positioned well. The percentage of the explained variance ratio is $82.2 \%$ and the factors successfully explain the items. The six factors were named as "Emotional Awareness" (F1), "Attention Regulation" (F2), "Body Listening" (F3), "Not-Distracting" (F4), "Trusting"(F5) and NotWorrying (F6), respectively.

Table 2 shows the descriptive statistics of the mean factor scores and internal consistency results of the MAIA 2 scales. The Cronbach Alpha values of the six factors were acceptable since they are greater than our threshold $(a>0.60)$. 


\begin{tabular}{|c|c|c|c|c|c|c|c|}
\hline Item & F1 & F2 & F3 & F4 & F5 & F6 & Communalities \\
\hline $\mathrm{i} 23$ & 0.994 & & & & & & 0.999 \\
\hline $\mathrm{i} 24$ & 0.994 & & & & & & 0.999 \\
\hline $\mathrm{i} 25$ & 0.994 & & & & & & 0.999 \\
\hline i26 & 0.994 & & & & & & 0.999 \\
\hline i27 & 0.994 & & & & & & 0.999 \\
\hline $\mathrm{i} 28$ & 0.994 & & & & & & 0.999 \\
\hline $\mathrm{i} 31$ & 0.994 & & & & & & 0.999 \\
\hline $\mathrm{i} 30$ & 0.994 & & & & & & 0.999 \\
\hline i13 & & 0.841 & & & & & 0.788 \\
\hline i14 & & 0.836 & & & & & 0.796 \\
\hline i2 & & 0.765 & & & & & 0.606 \\
\hline i3 & & 0.763 & & & & & 0.615 \\
\hline i17 & & 0.738 & & & & & 0.634 \\
\hline i5 & & 0.710 & & & & & 0.583 \\
\hline i15 & & 0.645 & & & & & 0.462 \\
\hline i16 & & 0.562 & & & & & 0.426 \\
\hline i18 & & 0.558 & & & & & 0.356 \\
\hline i21 & & & 0.985 & & & & 0.980 \\
\hline $\mathrm{i} 22$ & & & 0.985 & & & & 0.980 \\
\hline $\mathrm{i} 33$ & & & 0.985 & & & & 0.980 \\
\hline i34 & & & 0.985 & & & & 0.980 \\
\hline i7 & & & & 0.944 & & & 0.931 \\
\hline $\mathrm{i} 32$ & & & & 0.889 & & & 0.852 \\
\hline i10 & & & & 0.889 & & & 0.852 \\
\hline i8 & & & & 0.864 & & & 0.829 \\
\hline i9 & & & & 0.864 & & & 0.809 \\
\hline i36 & & & & & 0.830 & & 0.858 \\
\hline i37 & & & & & 0.830 & & 0.858 \\
\hline i4 & & & & & 0.620 & & 0.524 \\
\hline i29 & & & & & 0.618 & & 0.654 \\
\hline i12 & & & & & & 0.973 & 0.978 \\
\hline i11 & & & & & & 0.971 & 0.977 \\
\hline
\end{tabular}

\section{Table 2. The internal consistency results of the MAIA 2 scale}

\begin{tabular}{|lccc|}
\hline Factors & $\bar{X}$ & $\boldsymbol{\sigma}$ & Alpha \\
\hline F1 & 4.721 & 1.335 & 1 \\
F2 & 4.415 & 0.704 & 0.848 \\
F3 & 3.802 & 0.951 & 1 \\
F4 & 5.047 & 0.752 & 0.922 \\
F5 & 4.678 & 0.340 & 0.631 \\
F6 & 2.155 & 1.050 & 0.998 \\
\hline
\end{tabular}

Figure 1 shows CFA results of the MAIA 2 scale factors. While implementing CFA, we excluded three items because of the insignificant and the poor loading $(\beta<0.40)$ results. Then, we repeated the analysis and obtained the final CFA model. All standardized loadings were positive and greater than 0.40 with significant results $(\mathrm{p}<0.05)$. $(\mathrm{r}=0.71, \mathrm{p}<0.05)$.

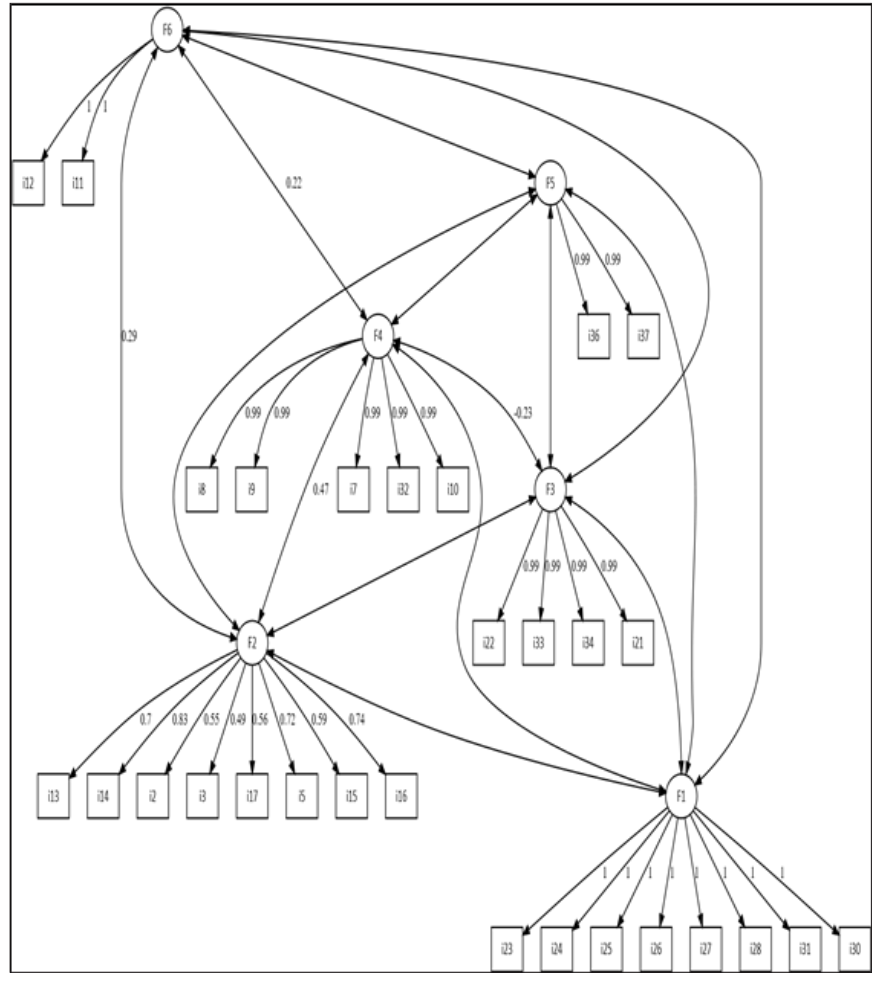

Figure 1. CFA results of MAIA 2 scale

Table 3 reports the goodness of fit index values of the CFA results for the MAIA 2 scale items. The fit index values showed that all the performance measures were perfect since GFI, AGFI, GFI, RFI, CFI were greater than 0.90 and much of them were remarkably close to 1 (Mulaik et al., 1989). The RMSEA was nearly zero and SRMR was extremely low. Lastly, $\chi 2 / \mathrm{sd}=0.179$ was lower than 2. All results pointed to the validity of the MAIA 2 scales.

Table 3. The goodness of fit index values for the CFA results for the MAIA 2

\begin{tabular}{|cccccccc|}
\hline$\chi^{2}$ & sd & GFI & AGFI & RFI & CFI & SRMR & RMSEA \\
\hline 64.816 & 362 & 0.966 & 0.959 & 0.991 & 1 & 0.039 & 0.000 \\
\hline
\end{tabular}

\section{DISCUSSION}

The aim of this study was to translate and adapt the MAIA 2 into the Turkish language, and to assess its psychometric properties in a Turkish speaking population.

The Turkish tool was tested in a sample of 403 participants aged between 20 and 62 years, from the provinces of Samsun, Turkey. This study was the first adaptation and validation of a self-report interoception assessment tool for the Turkey population. The adaptation was developed using a forward-backward translation, preserving the extension, format and the dimensional structure of the original scale. 
Due to the subjective self-report nature of the information collected by the MAIA (emotions and body sensations), achieving a conceptual equivalence (14) during the linguistic translation was a challenging process. Therefore, we adapted the MAIA 2 into Turkish using a systematic translation with the aid of a focus group. Three native Turkish speakers, fluent in English and familiar with the concepts of interoception and mindfulness, independently translated the 37 items of the English MAIA 2 version into Turkish.

We implemented exploratory factor analysis (EFA) and confirmatory factor analysis (CFA) to analyze the scale adoption process of the MAIA 2 scale items. We split the data into two parts (70:30 ratio) for exploring and validating the dimensions of the scale. In the first phase, we carried out EFA and reliability analysis based on the sub-dimensions. Then, we validated the factors using CFA. All the statistical analysis findings were obtained with R Project (10) using two R packages; psych (11) and lavaan (12).

The EFA favored a model with a factorial structure of eight dimensions with low factorial loading for items 1 and 6. Items 19 and 20 and 35 were removed because they did not contribute to the factors that they theoretically belong to. A new rotated factorial matrix was established for the 32-item scale. This matrix showed a factorial structure of six dimensions.

While the original scale includes eight factors, we found six dimensions with proper factor loadings and high communalities. Also, we observed that all the dimensions are rather reliable due to the satisfactory Cronbach's alpha values. In terms of the validity of the Turkish version of the MAIA scale, we obtained great fit indices and significant items on the sub-factors with CFA results. Consequently, Turkish version of MAIA 2 scale can be considered psychometrically valid.

In our study, unlike the original scale, different items created different scales. According to the analysis results in the study, it was determined that the items numbered $2,3,5,13-18$ were included in the scale of "emotional awareness". While only items 2 and 3 are located under the "noticing" in original scale, items numbered 13-15 is in "not worrying" and 16-18 is in "Attention Regulation". Therefore, considering the meanings of the items, it was decided that it would be more appropriate to collect this entire item group (2,3,13-18) under "Attention Regulation". According to the statistical analysis, the items numbered 21, 22, 33 and 34 that were included in the scale of "Attention Regulation" were gathered, in the scale "Body Listening" considering their meanings of those items.

The results suggest the necessity of making minor modifications (e.g., deletion or addition of items) to the original eight factor model to validate the MAIA scale in cross-cultural contexts (13-16). Since several studies suggested that conceptual and cultural differences may affect the construct of the MAIA (17-19). The findings imply that subjective aspects of interoceptive awareness are affected by the conceptual framework of a culture or population $(20,21)$

Future development of the MAIA 2 in the Turkish population should explore and provide evidence for convergent and divergent validity. Further studies with clinical and non-clinical populations, or samples with specific characteristics, are required to explore the differential performance of the items.

\section{CONCLUSION}

In this study, we attempted to adopt the MAIA 2 scale for healthcare workers in the Turkish population. Turkish version of the MAIA scale provides different structure from the original one. While the original version of the scale has eight factors, we obtained six factors. These factors were validated with CFA results with excellent model fit for six factors, namely "Emotional Awareness", "Attention Regulation", "Body Listening", "Not-Distracting", "Trusting" and Not-Worrying.

\section{ETHICAL DECLARATIONS}

Ethics Committee Approval: Ethical committee permission for the study was granted by Alanya Alaaddin Keykubat University Clinical Researches Ethics Committee (decision no: 20-3; date: 18.06.2020).

Informed Consent: All patients signed the free and informed consent form.

Referee Evaluation Process: Externally peer-reviewed.

Conflict of Interest Statement: The authors have no conflicts of interest to declare.

Financial Disclosure: The authors declared that this study has received no financial support.

Author Contributions: All of the authors declare that they have all participated in the design, execution, and analysis of the paper, and that they have approved the final version.

\section{REFERENCES}

1. Ceunen E, Vlaeyen JV, Diest IV. On the origin of interoception. Front Psychol 2016; 7: 1-17

2. Khalsa SS, Lapidus RC. Can interoception improve thepragmatic search for biomarkers in psychiatry? Front Psychol 2016; 7: 1-19

3. Mehling WE, Gopisetty V, Daubenmier J, Price CJ, Hecht FM, Stewart A. Body awareness: construct and self-report measures. PLoS ONE 2009; 4: e5614.

4. Mehling WE, Price C, Daubenmier JJ, Acree M, Bartmess E, Stewart A. The multidimensional assessment of interoceptive awareness (MAIA). PLoS ONE 2012; 7: e48230. 
5. Kabat-Zinn J. Full catastrophe living: Using the wisdom of your body and mind to face stress, pain, and illness $\left(15^{\text {th }}\right.$.). New York, NY: Random House 2008.

6. Multidimensional Assessment of Interoceptive Awareness. https: //osher.ucsf.edu/maia.

7. Mehling WE, Acree M, Stewart A, Silas J, Jones A. The multidimensional assessment of interoceptive awareness, version 2 (MAIA 2). PLoS ONE 2018; 12: e0208034.

8. Akgul A. Statistical analysis methods for medical research "spss applications". $3^{\text {rd }}$ ed. Ankara, Turkey: Emek Ofset; 2005.

9. Pierce AG. Measurement. In: Talbot LA (editor). Principles and Practice of Nursing Research. St. Louis, MO, USA: Mosby Year Book; 1995. pp. 265-291

10. R Core Team R: A language and environment for statistical computing. R Foundation for Statistical Computing, \#\# Vienna, Austria.2020 URL https: //www.R-project.org/.

11. Revelle W. Psych: Procedures for Personality and Psychological Research, Northwestern University, Evanston, Illinois, USA, https: //CRAN.R-project.org/package=psych Version=1.8.12.2018.

12. Rosseel Y. Lavaan: An R package for structural equation modeling and more. Version 0.5-12 (BETA). J Statis Softw 2012; 48: 1-36.

13.Sun CW, Pju K. Questionnaire translation and psychometric properties evaluation. SEGi University College 2009; 2: 45-51.

14. Holgado-Tello FP, Chacón-Moscoso S, Barbero-García I, et al. Polychoric versus Pearson correlations in exploratory and confirmatory factor analysis of ordinal variables. Qual Quant 2010; 441: 153.

15. Baranauskas M, Grabauskaitė A, Griškova-Bulanova I. Psychometric characteristics of Lithuanian version of Multidimensional Assessment of Interoceptive Awareness (MAIA). Neurologijos seminarai 2016; 20: 202-6.

16. Valenzuela-Moguillansky C, Reyes-Reyes A. Psychometric properties of the multidimensional assessment of interoceptive awareness (MAIA) in a Chilean population. Front Psychol 2015; 6: 120 .

17. Fujino $H$. Further validation of the Japanese version of the Multidimensional Assessment of Interoceptive Awareness. BMC Res Notes 2019; 12: 530 .

18. Wan-Suk Gim, Kyo-Lin Sim, Ok-Kyung Cho. Korean Multidimensional Assessment of Interoceptive Awareness (K-MAIA): Development and Validation. Korean J Stress Res 2016; 24: 177-90.

19. Fujino H. Further validation of the Japanese version of the Multidimensional Assessment of Interoceptive Awareness. BMC Res Notes 2019; 12: 530.

20.Wan-Suk Gim, Kyo-Lin Sim, Ok-Kyung Cho. Korean Multidimensional Assessment of Interoceptive Awareness (K-MAIA): Development and Validation. Korean J Stress Res 2016; 24: 177-90.

21.Shoji M, Mehling WE, Hautzinger M, Herbert BM. Investigating multidimensional interoceptive awareness in a Japanese Population: validation of the Japanese MAIA-J. Front Psychol 2018; 9: 1855. 